LA-UR-01-3251

Approved for public release; distribution is unlimited.

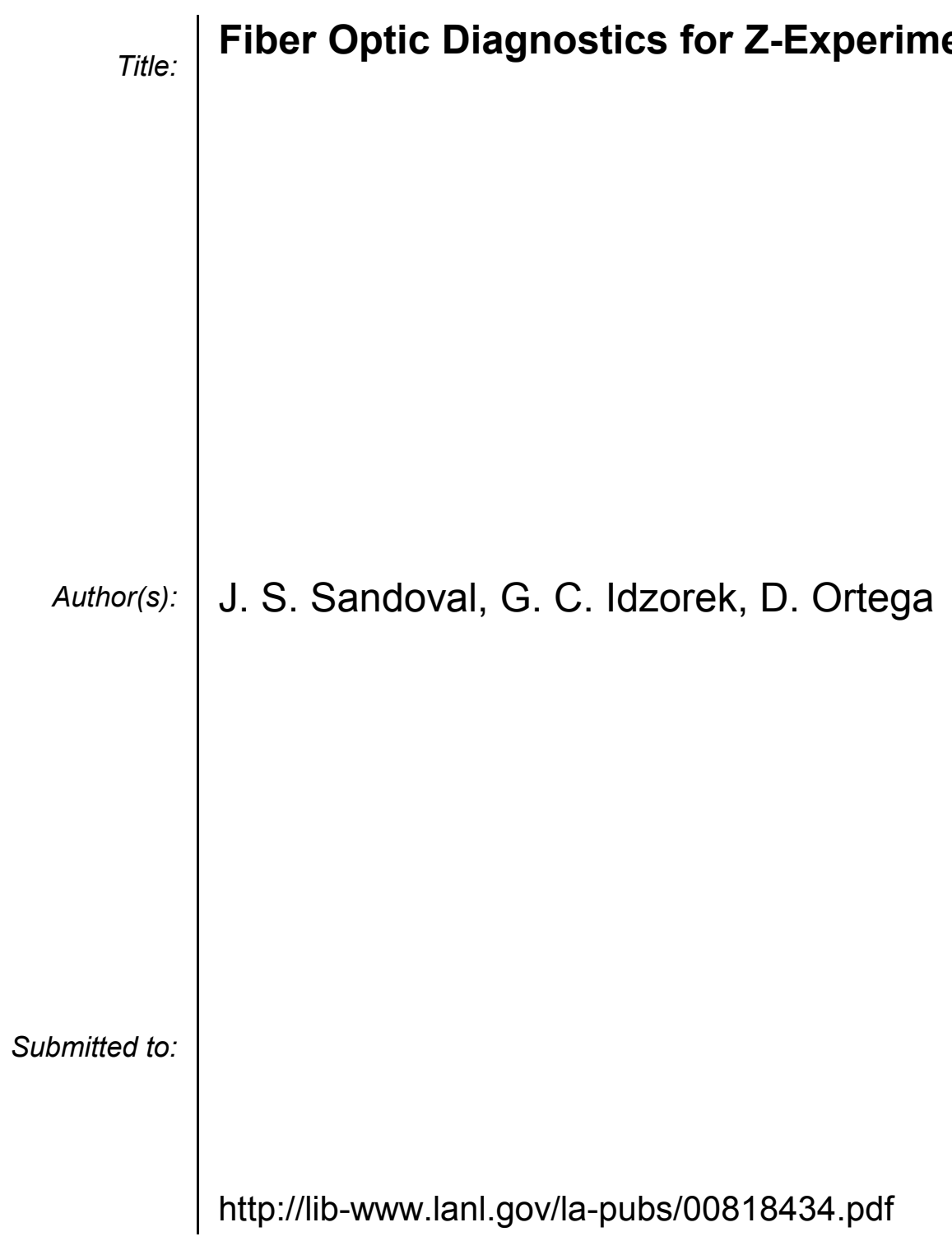

Los Alamos National Laboratory, an affirmative action/equal opportunity employer, is operated by the University of California for the U.S. Department of Energy under contract W-7405-ENG-36. By acceptance of this article, the publisher recognizes that the U.S. Government retains a nonexclusive, royaltyfree license to publish or reproduce the published form of this contribution, or to allow others to do so, for U.S. Government purposes. Los Alamos National Laboratory requests that the publisher identify this article as work performed under the auspices of the U.S. Department of Energy. Los Alamos National Laboratory strongly supports academic freedom and a researcher's right to publish; as an institution, however, the Laboratory does not endorse the viewpoint of a publication or guarantee its technical correctness. 


\title{
Fiber Optic Diagnostics for Z-Experiments
}

\author{
J. S. Sandoval, G. C. Idzorek, D. Ortega \\ Los Alamos National Laboratory $^{+}$ \\ Los Alamos, New Mexico, USA
}

\begin{abstract}
Quartz optical fiber provides an inexpensive, compact, radiation resistant, electrical noise immune shock diagnostic for our experiments on the Sandia National Laboratories Z-machine. The fiber end is positioned against a thin (10-30 micron thick) gold wall separating the fiber from the Z-machine radiation pulse. The radiation driven shock travels through the wall, striking the fiber and heating it to luminescent condition.
\end{abstract}

\section{Z-MACHINE OPERATION}

The Z-machine is constructed with 36 marx modules storing 11.4 MJ of electrical energy[1],[2]. The marxes are fired into pulse forming lines which synchronize and shorten the electric pulses. The 36 pulses converge onto a target located in the center of the machine. A 20 megampere pulse of current flows into a cylindrical target $20 \mathrm{~mm}$ diameter array of 300 individual 11.5 micron diameter tungsten wires. The resulting $\mathbf{J}$ X $\mathbf{B}$ forces implode the target which then stagnates on axis and generates a blackbody temperature of about $140 \mathrm{eV}$. This radiation pulse is coupled into our experiment packages and shocks the containment wall of the package and the fibers positioned along the outside of the wall.

\section{SHOCK SENSING WITH FIBER}

A shock pulse entering a fiber heats the fiber to luminescent conditions producing an intense light pulse with sub-nanosecond rise time. [3] The shock heated fiber temperature can be estimated from Equation of State tables for quartz [4]. Typical shock pressures on Zexperiments are in the 3 megabar range which corresponds to about $25,000^{\circ}$ Kelvin. The high shock pressures on $\mathrm{Z}$ typically produce so much light that the signal must be attenuated at the recording instruments.

\section{SENSOR CONSTRUCTION}

The diagnostic fiber bundle consists of multiple single strand 100-micron core step-index fibers (Polymicro \#FVP-100-110-125) that are positioned in precision
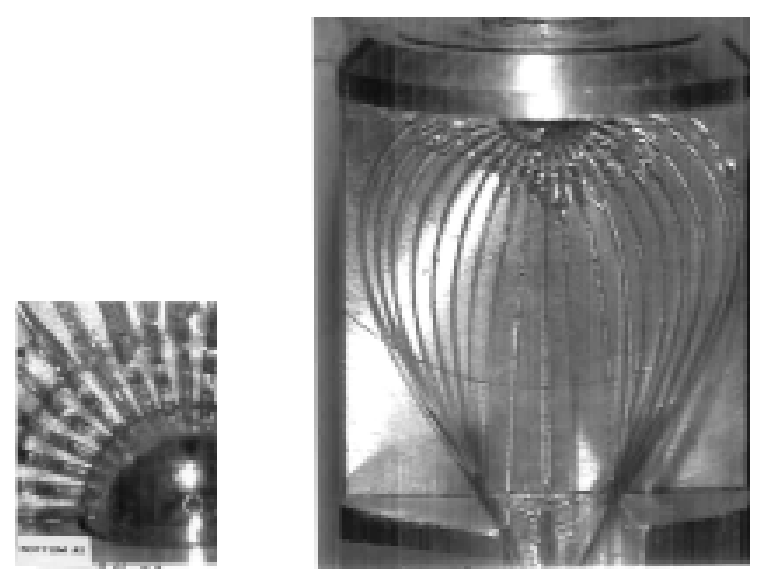

Figure 1. Fibers attached to experiment package

machined fixtures to accurately locate the polished fiber ends in intimate contact with the outside gold wall of our experiment packages. Figure 1. shows fibers radially arrayed around a $2.4 \mathrm{~mm}$ diameter hemispherical experiment package. The enlarged photo on the left shows the radial positioning slot machined into an aluminum mounting fixture. A small amount of UV curing epoxy is placed in slot and the fiber is positioned against the gold surface and held while the epoxy is cured.

The fiber bundle is constructed as continuous fibers from the experiment package through the z-machine

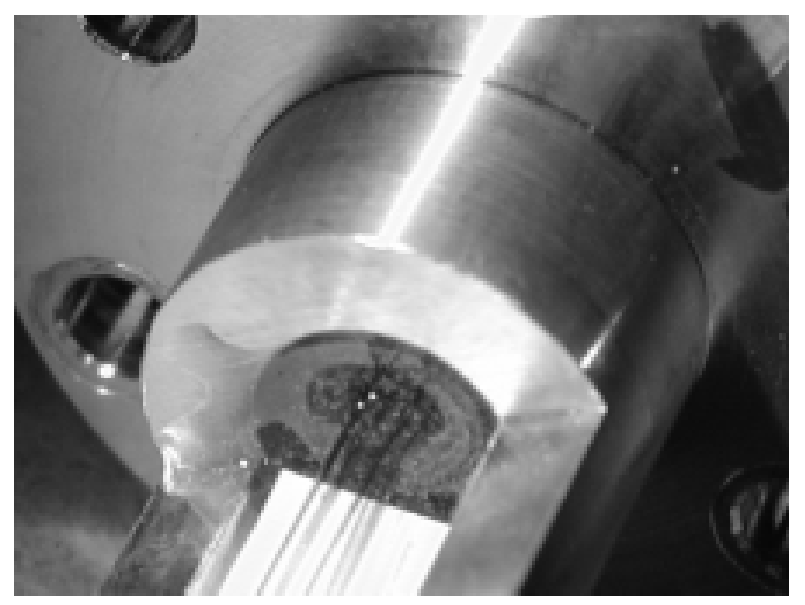

Figure 2. Epoxy vacuum sealed fibers

vacuum wall to a patch panel external to the machine. By placing all the fiber connections outside the vacuum

\footnotetext{
${ }^{+}$Los Alamos National Laboratory is operated by the University of California for the U. S. Department of Energy under contract W-7405-ENG-36.
} 
vessel truobleshooting is simplified and connection reliability is improved over connections made inside the explosion-debris coated vacuum chamber. Figure 2. shows the vacuum seal design. Bare fiber is threaded

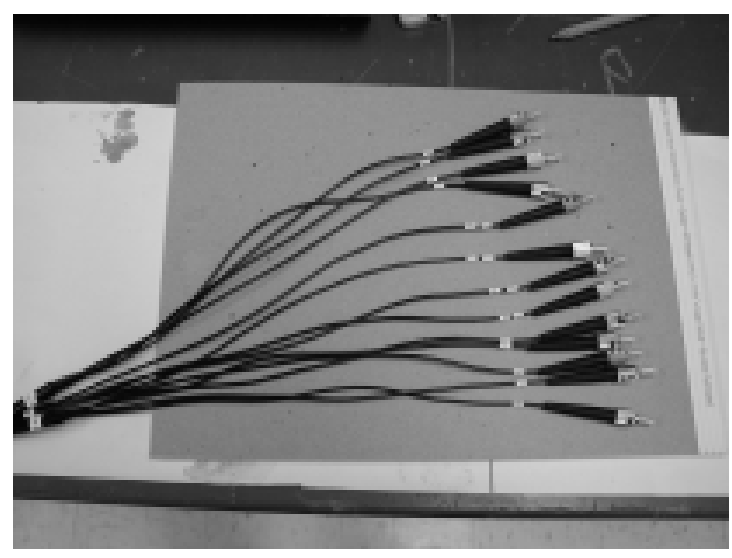

Figure 3. Fiber termination

through an aluminum housing and vacuum sealed using a slow cure epoxy (Hardman \#04006). Fibers external to the vacuum are individually sheathed in protective polyvinyl tubing and terminated with type ST fiber connector as shown in Figure 3. After termination the fiber bundle is checked for continuity using a laser pointer. The connector ends of the fiber are polished by using a 15 micron $\mathrm{SiC}$ grit, then a 3 micron $\mathrm{Al}_{2} \mathrm{O}_{3}$, followed by 0.3 micron $\mathrm{Al}_{2} \mathrm{O}_{3}$. The polish is checked using a fiber microscope and repeated if necessary, a good polish appears as a dark uniform circle of glass. The target end of the fiber is polished by inserting the fiber into a connector and holding it by hand flush with

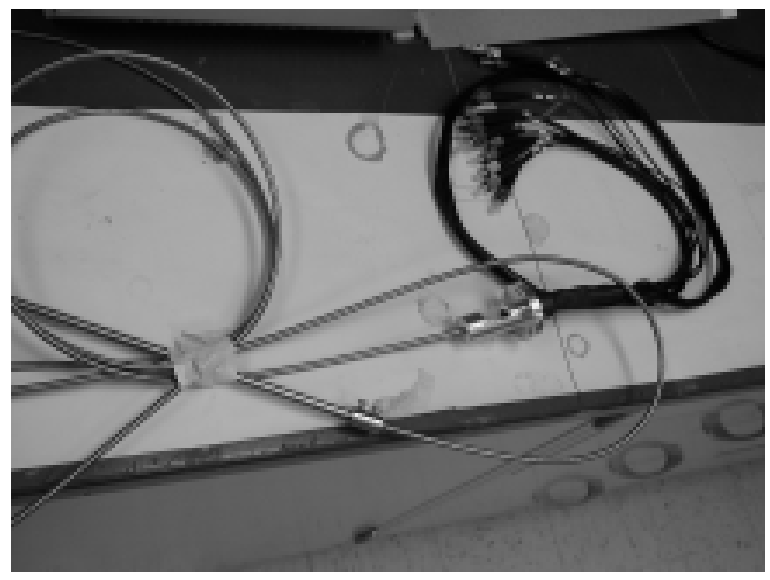

Figure 4. Completed fiber assembly

the connector end then gently rubbing it with a sheet of polishing grit. The fiber end is then examined under a microscope to ensure the fiber is square and without chips and cracks. The completed fiber assembly shown in Figure 4. is vacuum leak checked to $1 \times 10^{-9} \mathrm{~atm}-\mathrm{cc} / \mathrm{s}$ leak rate. After labeling, an optical TDR is used to measure the optical length of the fibers with 10 ps resolution.
Inside the vacuum vessel the fibers are enclosed in a flexible steel radiation shield from the experiment to the vacuum bulkhead fitting. After assembly and testing the fiber assembly is sent to the target fabrication group for attachment of the experiment package.

\section{DATA RECORDING}

The visible light pulses generated during the experiment are recorded by optical streak cameras and also sent to optical-to-electrical converters for recording on standard electronic digitizers. Figure 5 shows a streak record of a stepped witness plate experiment. The plate thickness were 100 and 200 microns of gold.

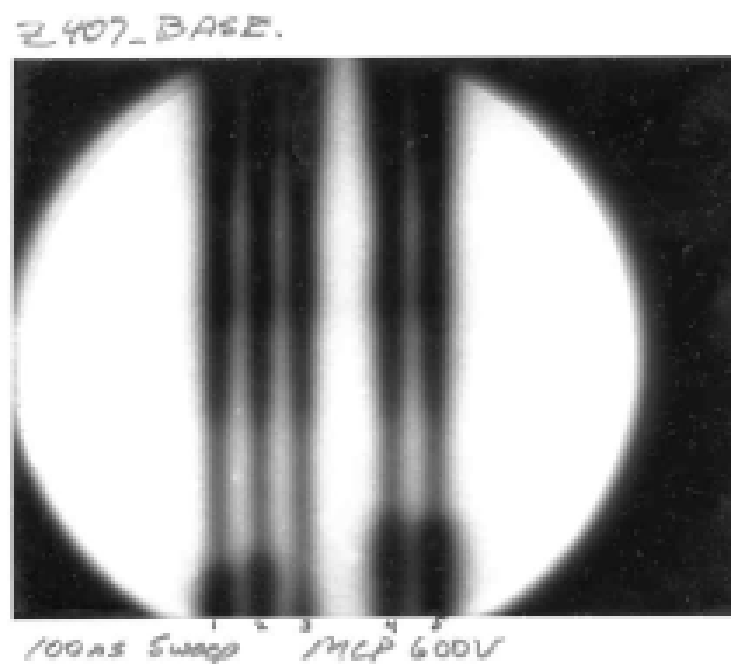

Figure 5. Streak record of stepped witness plate.

The first two fibers on the left side were mounted on the 200 micron step and the two rightmost fibers were mounted on the 100 micron step. Time flows from top to bottom. The center fiber was not in contact with the witness plate and served as a background signal. The background was mainly due to high energy photons producing Cerenkov light in the fibers. Even with this large background signal the difference in shock breakout time is clearly seen.

\section{V.SUMMARY}

The Z-machine provides a fast pulsed high temperature source for radiation flow experiments.

Quartz fiber shock sensors provide a simple, noninvasive way to measure the radiation flow inside a thin walled experiment package. Multiple experiments can be fielded simultaneously all driven by an identical radiation source thereby eliminating the often difficult corrections to results obtained from multiple shots with a single experiment. 
By using an optical streak camera to record up to 100 fibers on an experiment cross timing errors are eliminated.

\section{REFERENCES}

[1] R. B. Spielman, et al., "Pulsed Power Performance of PBFA-Z," in Proc. of the $11^{\text {th }}$ IEEE International Pulsed Power Conference, 1997, pp.709-714

[2] R. B. Spielman, et al., "Tungsten wire-array Z-pinch experiments at $200 \mathrm{TW}$ and 2 MJ," Physics of Plasmas, vol. 5, (no. 5), pp. 2105-2111, May 1998

[3] L. R. Veeser, et al., "Sensing of high pressure by shock heating quartz optical fibers," in Proc. of SPIE, Vol. 838, 1987, pp. 60-68

[4] G. A. Lyzenga, T. J. Ahrens, "Shock Temperarure Measurements in $\mathrm{Mg}_{2} \mathrm{SiO}_{4}$ and $\mathrm{SiO}_{2}$ at $\mathrm{High}$ Pressures," Geo. Res. Lett. vol. 7, 1980, pg. 141 